hep-th/0203233

\title{
Baryon Masses and Wilson Loops for Fractional D3-Branes on the Resolved Conifold
}

\author{
Shijong Ryang \\ Department of Physics \\ Kyoto Prefectural University of Medicine \\ Taishogun, Kyoto 603-8334 Japan \\ ryang@koto.kpu-m.ac.jp
}

\begin{abstract}
We study the IR dynamics of the type IIB supergravity solution describing $N$ D3branes and $M$ fractional D3-branes on the resolved conifold. The baryon mass and the tension of domain wall in the dual gauge theory are evaluated and compared with those for the deformed conifold. The IR behavior of the solution for the general conifold is also discussed. We show that the area law behavior of the Wilson loop is attributed to the existence of the locus in the IR where the D3-brane charge vanishes.
\end{abstract}

March, 2002 


\section{Introduction}

The original AdS/CFT correspondence [1], 2, [0] relating the supergravity and the strongly coupled superconformal gauge theory has been extended to the systems with less supersymmetry [4, 5, 6, 7, 8, 9, 10] and the non-conformal systems [11, 12, 13, 14]. If the $N$ D3-branes are placed at the apex of a six-dimensional cone whose base is a five-dimensional Einstein manifold $X_{5}$, supersymmetry is partially broken and the type IIB string theory on $A d S_{5} \times X_{5}$ is considered to be dual to the low energy limit of the worldvolume theory on the D3-branes at the conifold singularity, which is a $\mathcal{N}=1$ superconformal gauge theory with $S U(N) \times S U(N)$ gauge group. Breaking conformal invariance can be performed by placing the additional $M$ fractional D3-branes at the singularity. The fractional D3-branes are thought of as D5-branes wrapped over collapsed 2-cycles at the singularity and modify gauge group to $S U(N+M) \times S U(N)$ [12, [13]. The conformal invariance is broken so that the relative gauge coupling runs logarithmically and the supergravity equations are solved to leading order in $M / N$ [13] and to all orders [14]. The solution has a naked singularity at small radius. The appearance of naked singularities in the gravitational duals of the non-conformal gauge theories with reduced supersymmetry is rather common phenomena [15, [16, 17].

In Ref. 18 the solution without the conical singularity has been constructed via the deformation of the conifold where the apex is replaced by a $S^{3}$. The decrease of the D3brane charge is identified with the repeated chain of Seiberg-duality transformations. In this solution there appear no naked singularities in the IR. At the IR end of the duality cascade the theory becomes a $\mathcal{N}=1 S U(M)$ gauge theory which confines with chiral symmetry breaking. Based on this theory the spectrum of glueball masses has been calculated [19] and the gluino condensation as well as the Wilson loop have been investigated [20, 21]. The alternative approach for removing the naked singularity is to put the system at high temperature, which leads to the restoration of chiral symmetry [22, 23, 24]. On the other hand the conical singularity is removed by the resolution of the conifold where the apex is replaced by a $S^{2}$ [25, 26] or by the generalization of the conifold [26]. In the system of $N$ regular D3-branes and $M$ fractional D3-branes on the resolved conifold or the general conifold a naked repulson-type singularity emerges far in the IR and is located behind the radius where the R-R 5-form flux vanishes. For the system of only the $N$ regular D3-branes on the resolved conifold or the general conifold, the Wilson loop for a quark anti-quark interaction is studied [26].

We will further analyze the solution of $N$ D3-branes as well as $M$ fractional D3-branes on the resolved conifold or the general conifold to extract IR properties for the baryon mass, the tension of domain wall and the tension of fractional D3-brane. The Wilson loop for the dual non-conformal gauge theory will be shown to take the area law behavior which is associated with the existence of the locus in the IR where the R-R 5-form flux vanishes. 


\section{Baryon masses and domain walls}

Let us write the metric of the type IIB supergravity solution representing $N$ regular D3branes and $M$ fractional D3-branes on the general resolved conifold [26]

$$
d s_{10}^{2}=h^{-1 / 2}(\rho) d x^{\mu} d x^{\mu}+h^{1 / 2}(\rho) d s_{6}^{2},
$$

where the six-dimensional metric on the general resolved conifold is given by

$$
d s_{6}^{2}=\kappa^{-1}(\rho) d \rho^{2}+\frac{1}{9} \kappa(\rho) \rho^{2} e_{\psi}^{2}+\frac{1}{6} \rho^{2}\left(e_{\theta_{1}}^{2}+e_{\phi_{1}}^{2}\right)+\frac{1}{6}\left(\rho^{2}+6 a^{2}\right)\left(e_{\theta_{2}}^{2}+e_{\phi_{2}}^{2}\right)
$$

with the resolution parameter $a$ and

$$
\kappa(\rho)=\frac{1+\frac{9 a^{2}}{\rho^{2}}-\frac{b^{6}}{\rho^{6}}}{1+\frac{6 a^{2}}{\rho^{2}}}, e_{\psi}=d \psi+\sum_{i=1}^{2} \cos \theta_{i} d \phi_{i}, e_{\theta_{i}}=d \theta_{i}, e_{\phi_{i}}=\sin \theta_{i} d \phi_{i}, i=1,2 .
$$

The length scale $b$ is also introduced as a generalization parameter to smoothen the curvature singularity of the original conifold metric. There appears an asymmetry between the two $S^{2}$ parts of the resolved conifold $(a \neq 0, b=0)$, while there is no asymmetry for the general conifold $(a=0, b \neq 0)$. In the resolved conifold near the apex the $S^{3}\left(\psi, \theta_{1}, \phi_{1}\right)$ part of the metric shrinks to zero size and the $S^{2}\left(\theta_{2}, \phi_{2}\right)$ part remains finite with a radius $a$. The $M$ D5-branes wrapped over the 2-cycle that are identified with the fractional D3-branes serve as sources of the magnetic R-R 3-form flux to give

$$
F_{3}=P e_{\psi} \wedge\left(e_{\theta_{2}} \wedge e_{\phi_{2}}-e_{\theta_{1}} \wedge e_{\phi_{1}}\right)
$$

with $P \sim M \alpha^{\prime}$. The type IIB equations are satisfied by the NS-NS 2-form potential and the R-R 5-form flux

$$
\begin{aligned}
B_{2} & =f_{1}(\rho) e_{\theta_{1}} \wedge e_{\phi_{1}}+f_{2}(\rho) e_{\theta_{2}} \wedge e_{\phi_{2}} \\
F_{5} & =\mathcal{F}+* \mathcal{F}, \mathcal{F}=K(\rho) e_{\psi} \wedge e_{\theta_{1}} \wedge e_{\phi_{1}} \wedge e_{\theta_{2}} \wedge e_{\phi_{2}}
\end{aligned}
$$

together with the warp function $h$ determined from the trace of the Einstein equation as

$$
h^{\prime}=-\frac{108 g_{s} K}{\rho^{5} \kappa \Gamma},
$$

where $K=Q+P\left(f_{1}-f_{2}\right), Q \sim N \alpha^{\prime 2}$ and $\Gamma=\left(\rho^{2}+6 a^{2}\right) / \rho^{2}$. Following Ref. [21] we can fix the normalization as

$$
Q=\frac{1}{4} \pi N \alpha^{\prime 2}, P=\frac{1}{4} M \alpha^{\prime}
$$

For the general conifold the symmetric solution is given by

$$
f_{1}=-f_{2}=\frac{1}{2} g_{s} P \ln \left(\bar{\rho}^{6}-1\right)+f_{0}, K=Q+g_{s} P^{2} \ln \left(\bar{\rho}^{6}-1\right)+2 P f_{0}
$$

with $\bar{\rho}=\rho / b \geq 1$. In the following we will devote ourselves to the case that the number of fractional D3-branes is much smaller than that of regular D3-branes such that $g_{s} N \gg\left(g_{s} M\right)^{2}$ 
under the large $g_{s} N, g_{s} M$ supergravity regime. In this case the integration constant $f_{0}$ can be neglected. The warp function $h$ obtained by substituting $K$ of (8) into (6) and integrating, shows the standard conifold behavior

$$
h=h_{0}+\frac{27}{\rho^{4}}\left[g_{s} Q+6\left(g_{s} P\right)^{2}\left(\ln \bar{\rho}+\frac{1}{4}\right)\right]
$$

in the large $\rho$ region for the string metric, while it gives the short distance behavior

$$
h=h_{0}-\frac{18 g_{s} Q}{b^{4}} \ln \left(\bar{\rho}^{2}-1\right)-\frac{9\left(g_{s} P\right)^{2}}{b^{4}} \ln ^{2}\left(\bar{\rho}^{2}-1\right) .
$$

On the other hand the asymmetric solution for the resolved conifold is expressed as [25]

$$
\begin{aligned}
& f_{1}=\frac{3}{2} g_{s} P \ln \left(\rho^{2}+9 a^{2}\right)+f_{10}, f_{2}=\frac{1}{6} g_{s} P\left(\frac{36 a^{2}}{\rho^{2}}-\ln \left[\rho^{16}\left(\rho^{2}+9 a^{2}\right)\right]\right)+f_{20}, \\
& K=Q-\frac{1}{3} g_{s} P^{2}\left(\frac{18 a^{2}}{\rho^{2}}-\ln \left[\rho^{8}\left(\rho^{2}+9 a^{2}\right)^{5}\right]\right)+P\left(f_{10}-f_{20}\right),
\end{aligned}
$$

where $f_{10}$ and $f_{20}$ are the constants of integration. Alternatively if we choose the integration constants $\rho_{1}, \rho_{2}$ instead of $f_{10}, f_{20}$ as the following

$$
f_{1}=\frac{3}{2} g_{s} P \ln \frac{\rho^{2}+9 a^{2}}{\rho_{1}^{2}}, f_{2}=\frac{1}{6} g_{s} P\left(\frac{36 a^{2}}{\rho^{2}}-\ln \frac{\rho^{16}\left(\rho^{2}+9 a^{2}\right)}{\rho_{2}^{18}}\right),
$$

we have

$$
K=Q-\frac{1}{3} g_{s} P^{2}\left(\frac{18 a^{2}}{\rho^{2}}-\frac{9}{2} \ln \frac{\rho^{2}+9 a^{2}}{\rho_{1}^{2}}-\frac{1}{2} \ln \frac{\rho^{16}\left(\rho^{2}+9 a^{2}\right)}{\rho_{2}^{18}}\right) .
$$

The large $\rho(\rho \gg 3 a)$ behavior of $h$ is also the same as the warp function for the standard conifold

$$
h=h_{0}+\frac{27}{\rho^{4}}\left[g_{s} Q+\frac{3}{2}\left(g_{s} P\right)^{2}\left(\ln \frac{\rho^{2}}{\rho_{1}^{2}}+\ln \frac{\rho^{2}}{\rho_{2}^{2}}+1\right)\right]
$$

and in the IR region $(\rho \ll 3 a) h$ shows the power behavior

$$
h=h_{0}+\frac{6 g_{s} Q}{a^{2} \rho^{2}}-\frac{18\left(g_{s} P\right)^{2}}{\rho^{4}} .
$$

Hereafter we set $h_{0}=0$ to consider the near-core region. The warp function has a naked singularity at $\rho=\rho_{h}, \rho_{h}^{2}=3 g_{s} P^{2} a^{2} / Q$ and the R-R 5-form flux vanishes at $\rho=\rho_{K}, \rho_{K}=$ $\sqrt{2} \rho_{h}$. The inequality $\rho_{K}>\rho_{h}$ is also seen in the general conifold where the naked singularity and the zero-charge locus $(K=0)$ are located at $\overline{\rho_{h}} \approx 1+e^{-2 Q /\left(g_{s} P^{2}\right)} / 2$ and $\overline{\rho_{K}} \approx 1+$ $e^{-Q /\left(g_{s} P^{2}\right)} / 6$ respectively.

Now we are ready to consider a regular D3-brane probe in the background of $N$ D3branes and $M$ fractional D3-branes on the general resolved conifold. The relevant D3-brane probe action is given by

$$
S=-T_{D 3} \int d^{4} \sigma e^{-\phi} \sqrt{-\operatorname{det}\left(g_{a b}+\mathcal{F}_{a b}\right)}+\mu_{D 3} \int C_{4},
$$


where $T_{D 3}$ and $\mu_{D 3}$ are the tension and basic $\mathrm{R}$-R charge of the D3-brane, and $\mathcal{F}_{a b}=$ $B_{a b}+2 \pi \alpha^{\prime} F_{a b}$. Here $g_{a b}$ and $B_{a b}$ are the pulls-back of the ten-dimensional metric and the NS-NS 2-form potential. Let us assume that the probe D3-brane is parallel to the source of $N$ D3-branes and $M$ fractional D3-branes and extends to the $x^{1}, x^{2}, x^{3}$ directions with the (infinite) volume $V_{3}$. Taking the Hodge dual of the expression $\mathcal{F}$ in (5) we have

$$
* \mathcal{F}=\frac{108 K}{h^{2} \rho^{5} \kappa \Gamma} d \rho \wedge d x^{0} \wedge d x^{1} \wedge d x^{2} \wedge d x^{3} .
$$

In view of $F_{5}=d C_{4}+B_{2} \wedge F_{3}$ together with (4), (5) the $\left(\rho, x^{0}, x^{1}, x^{2}, x^{3}\right)$ component of $B_{2} \wedge F_{3}$ vanishes so that the expression (17) yields

$$
C_{4}=\frac{1}{g_{s} h} d x^{0} \wedge d x^{1} \wedge d x^{2} \wedge d x^{3}
$$

where the Eq. (6) has been used. The substitution of (18) and the background solution into the probe action (16) in the static gauge leads to

$$
S=-V_{3} \int d t\left(\frac{T_{D 3}}{h}-\frac{\mu_{D 3}}{g_{s} h}\right) .
$$

This vanishing implies that the gravitational attractive force between a probe D3-brane and the source expressed as

$$
F=-T_{D 3} V_{3} \frac{\partial}{\partial \rho}\left(\frac{1}{h}\right)=-T_{D 3} V_{3} \frac{108 g_{s} K}{h^{2} \rho^{5} \kappa \Gamma}
$$

cancels out against the repulsive electric force between the R-R charges with the same sign. The gravitational attractive force (20) vanishes at the zero-charge locus and diverges at the naked singularity and becomes repulsive between the two locations. If we consider an anti-D3-brane probe the cancellation does not happen and the total interaction becomes attractive.

There is the other configuration that a probe D3-brane wraps the 3-cycle of the general resolved conifold. The wrapped D3-brane is considered to play the role of a baryon vertex [12]. Since the relevant worldvolume action has no contribution from the Wess-Zumino term, the wrapped D3-brane will feel the attractive force only. For the resolved conifold a D3-brane can wrap over two kinds of 3-cycle associated with constant $\left(\theta_{1}, \phi_{1}\right)$ or $\left(\theta_{2}, \phi_{2}\right)$. For the probe D3-brane wrapped over $S^{3}\left(\psi, \theta_{1}, \phi_{1}\right)$ of the resolved conifold we substitute the classical solution describing a collection of $N$ D3-branes and $M$ fractional D3-branes into the probe action in the static gauge to obtain a baryon mass

$$
M_{B}=\frac{8 \pi^{2}}{9} T_{D 3 \sqrt{\kappa_{a}}} \rho\left[h \rho^{4}+\left(6 f_{1}\right)^{2}\right]^{1 / 2}
$$

with $\kappa_{a}=\left(\rho^{2}+9 a^{2}\right) /\left(\rho^{2}+6 a^{2}\right)$, which includes the nontrivial contribution from the NSNS $B_{2}$ field. The baryon mass, that is, the total energy of the static wrapped D3-brane is a product of D3-brane tension, area of $S^{3}\left(\psi, \theta_{1}, \phi_{1}\right)$, the gravitational potential $\sqrt{-g_{00}}$ 
and so on. The variable $\rho$ represents the position of the wrapped D3-brane, which feels an attractive radial force $-\partial M_{B} / \partial \rho$ which corresponds to (20), and the other forces exerted by $M$ fundamental strings attatched on itself. In the UV regin $(\rho \rightarrow \infty)$ the baryon mass is expressed as

$$
M_{B}=\frac{\rho}{2 \pi \alpha^{\prime} g_{s}}\left[\frac{1}{3} \pi g_{s} N_{e f f}+\left(g_{s} M\right)^{2} \ln ^{2} \rho\right]^{1 / 2}
$$

with the effective D3-brane charge, $N_{\text {eff }}=N+(3 / 2 \pi) g_{s} M^{2} \ln \rho$. This expression may be compared with the baryon mass $M_{B}=\rho N /\left(8 \pi \alpha^{\prime}\right)$, that is linear in $N$, for a D5-brane wrapped over $S^{5}$ in the $A d S_{5} \times S^{5}$ background 27, 28]. The attractive gravitational force due to the $N$ D3-branes is constant for the latter, while that for the former shows a logarithmic increase which is dominantly attributed to the NS-NS $B_{2}$ field. On the other hand in the IR region the baryon mass at the zero-charge locus $\rho=\rho_{K}, \rho_{K}=g_{s} P a \sqrt{6 / g_{s} Q}$ is shown to scale as

$$
M_{B} \sim M \frac{\rho_{K}}{\alpha^{\prime}} .
$$

Compared to the baryon mass (21) we have the mass of wrapped D3-brane

$$
M_{D 3}=\frac{8 \pi^{2}}{9} T_{D 3} \sqrt{\kappa_{a}}\left(h \rho^{4}\right)^{1 / 4}\left[h \rho^{4}+\left(6 f_{1}\right)^{2}\right]^{1 / 2},
$$

which does not include the gravitational potential $\sqrt{-g_{00}}$. For the conifold case $(a=0)$ with no fractional D3-branes this mass is independent of $\rho$ and leads through the normalization (7) to the conformal dimension $\Delta=3 N / 4$ of the corresponding baryonic vertex operator in the dual conformal field theory [12]. From (24) we note that the mass of wrapped D3-brane localized at a constant $\left(\theta_{2}, \phi_{2}\right)$ vanishes at the naked singularity $\rho=\rho_{h}$. Although there is a repulsive component of the radial force $-\partial M_{B} / \partial \rho$ in the region $\rho_{h}<\rho<\rho_{K}$, the $\rho$ dependent attractive force prevails over it. The wrapped D3-brane is always pulled toward the apex and then becomes massless at the naked singularity. This behavior is compared with the $M=0$ conifold case where the wrapped D3-brane with the constant mass is pulled by a constant attractive force because of the $\rho$-independence of $h \rho^{4}$ in (21). Here we consider the other 3 -cycle defined by the subspace at a constant value of $\left(\theta_{1}, \phi_{1}\right)$ in the metric. The D3-brane wraps over $\left(\psi, \theta_{2}, \phi_{2}\right)$ coordinates to yield a baryon mass

$$
M_{B}=\frac{8 \pi^{2}}{9} T_{D 3} \sqrt{\kappa_{a}} \rho\left[h \rho^{4}+\left(6 f_{2}\right)^{2}\right]^{1 / 2}
$$

whose large $\rho$ behavior is the same as (22). At the IR point specified by $\rho=\rho_{K}$ it takes

$$
M_{B} \sim \frac{a}{\sqrt{g_{s} Q} \alpha^{\prime 2}}\left(Q^{2}+\frac{g_{s}^{2} P^{4}}{2}\right)^{1 / 2}
$$

which scales as

$$
M_{B} \sim M \frac{\sqrt{g_{s} N} a}{g_{s} M \alpha^{\prime}}\left(1+\frac{\left(g_{s} M\right)^{4}}{32 \pi^{2}\left(g_{s} N\right)^{2}}\right)^{1 / 2} .
$$


This result shows a factor of $M$ times the 't Hooft scalings $g_{s} N$ and $g_{s} M$. For small number of fractional D3-branes the baryon mass is approximately expressed as

$$
M_{B} \sim M \frac{a^{2}}{\rho_{K} \alpha^{\prime}}
$$

because the NS-NS 2-form potential yields the dominant contribution.

In the general conifold the baryon mass for the D3-brane wrapped over $S^{3}\left(\psi, \theta_{1}, \phi_{1}\right)$ is also estimated as

$$
M_{B} \sim \frac{Q b}{g_{s} P \alpha^{2}} e^{-Q / 2 g_{s} P^{2}}
$$

where the exponential damping arises from the factor $\sqrt{\kappa_{b}}$ with $\kappa_{b}=\left(\rho^{6}-b^{6}\right) / \rho^{6}$ and the NS-NS flux contribution, $\left(6 f_{1}\right)^{2}$ has the same order as the gravitational contribution through the warp factor, $h \rho_{K}^{4}$. This expression also shows the expected scaling

$$
M_{B} \sim M \frac{g_{s} N b}{\left(g_{s} M\right)^{2} \alpha^{\prime}} e^{-2 \pi g_{s} N /\left(g_{s} M\right)^{2}}
$$

which has some resemblance to (27) up to the exponential factor.

For comparison let us return to the standard conifold. The warp function is given by (9) with $\ln (\rho / b)$ replaced by $\ln \left(\rho / \rho_{0}\right)$ where $\rho_{0}$ is introduced as a constant of integration. The NS-NS 2-form potential and the charge function $K(\rho)$ are provided by (12), (13) with $a=0$ and $\rho_{1}=\rho_{2} \equiv \rho_{0}$. The baryon mass is also evaluated at the zero-charge locus $\rho_{K}=e^{-Q / 6 g_{s} P^{2}} \rho_{0}$ as

$$
M_{B} \sim \frac{\rho_{0}}{g_{s} P \alpha^{\prime 2}} e^{-Q / 6 g_{s} P^{2}}\left(Q^{2}+\frac{g_{s}^{2}(3 P)^{4}}{2}\right)^{1 / 2}
$$

whose leading part scales as

$$
M_{B} \sim M \frac{g_{s} N \rho_{0}}{\left(g_{s} M\right)^{2} \alpha^{\prime}} e^{-2 \pi g_{s} N / 3\left(g_{s} M\right)^{2}}
$$

Although this expression is similar to (30), the exponential damping factor is here produced by the linear factor $\rho_{K}$ and the NS-NS flux gives the main contribution. The dimensions of baryon masses are specified by the resolution parameter $a / \alpha^{\prime}$ in (23), (27) for the resolved conifold and similarly by the generalization parameter $b / \alpha^{\prime}$ in (30) for the general conifold. They are contrasted to the factor $\rho_{0} / \alpha^{\prime}$ in (32) for the conifold. The factor $\rho_{0}$ is provided as an integration constant, whereas the emergences of $a, b$ in the baryon mass at the IR locus indicate the dimensional transmutation.

In the deformed conifold [18, 21] the radial parameter $\tau$ related with the radial coordinate $\rho$ as $\rho^{3} \sim \epsilon^{2} e^{\tau}$ can be made zero, where $\rho$ approaches to $\epsilon^{2 / 3}$ and the $S^{2}$ part of the metric shrinks to zero while the radius of $S^{3}$ part approaches to finite value that is of order $\sqrt{g_{s} M}$. The baryon mass for the D3-brane wrapped over the finite $S^{3}$ part is estimated as $M_{B} \sim m M$ where $m \sim \epsilon^{2 / 3} / \alpha^{\prime}$ is a parameter setting the 4-dimensional mass scale. This estimation for the deformed conifold is similar to that for the resolved conifold although there is some 
difference of situation. The expression (23) is produced for the D3-brane wrapped over the shrinking $S^{3}\left(\psi, \theta_{1}, \phi_{1}\right)$ whereas (28) is for the D3-brane over the $S^{3}\left(\psi, \theta_{2}, \phi_{2}\right)$ whose $S^{2}\left(\theta_{2}, \phi_{2}\right)$ part has a finite radius near the apex. The parameter $\rho_{K}$ that is proportional to the resolution parameter a makes a role of setting the 4-dimensional mass scale, while the extra parameter $b$ makes the same role in the general conifold. For the deformed conifold the theory at $\tau=0$ provides the $S U(M)$ gauge theory with no matter through the chain of Seiberg dualites. Analogously for the general or resolved conifold the far IR locus is so specified by the zero-charge locus that the regular D3-branes disappear and only the $M$ fractional D3-branes remain. Hence the supergravity theory at the zero-charge locus for the general or resolved conifold is considered to be dual to the $S U(M)$ gauge theory. The obtained baryon masses are shown to be linear in $M$ as expected from this view point.

Here we also analyze the domain walls that are regarded as D5-branes wapping the $S^{3}$ and spanning the $x^{0}, x^{1}, x^{2}$ directions for the resolved conifold. Far in the IR the effective $S U(M)$ gauge theory has the inequivalent vacuum states between which the domain wall interpolates. The tension of a D5-brane wrapped over $S^{3}\left(\psi, \theta_{1}, \phi_{1}\right)$ as well as $x^{0}, x^{1}, x^{2}$ coordinates at the IR locus $\rho=\rho_{K}$ is estimated as

$$
T_{D W}=\frac{8 \pi^{2}}{9} T_{D 5} \sqrt{\frac{\kappa_{a}}{h}} \rho_{K}\left[h \rho_{K}^{4}+\left(6 f_{1}\right)^{2}\right]^{1 / 2} \sim \frac{\rho_{K}^{3}}{g_{s} \alpha^{\prime 3}},
$$

whereas the tension for the wrapping over $S^{3}\left(\psi, \theta_{2}, \phi_{2}\right)$ is mainly given by

$$
T_{D W} \sim \frac{\rho_{K} a^{2}}{g_{s} \alpha^{\prime 3}} .
$$

The former behavior is compared with $T_{D W} \sim\left(\epsilon^{2 / 3} / \alpha^{\prime}\right)^{3} / g_{s}$ for the deformed conifold [18, 21]. Through the wrappings of a D5-brane and a D3-brane over $S^{3}\left(\psi, \theta_{1}, \phi_{1}\right)$ for the resolved conifold the tension of domain wall and the baryon mass, where the gravitational contribution is the same order as the $B_{2}$ contribution, show the same forms as those for the deformed conifold where the $B_{2}$ contribution is not taken into account. Hence $\rho_{K} / \alpha^{\prime}$ corresponds to $m \sim \epsilon^{2 / 3} / \alpha^{\prime}$. On the other hand in the wrapping over $S^{3}\left(\psi, \theta_{2}, \phi_{2}\right)$ for the resolved conifold the tension of domain wall and the baryon mass where the $B_{2}$ contribution is more dominant than the gravitational one, show the similar forms to those for the deformed conifold, but with some slight difference.

\section{Tensions of fractional D-branes}

We are concerned with the tensions of D5-brane wrapped over $S^{2}\left(\theta_{i}, \phi_{i}\right), i=1,2$. They can be also evaluated and shown not to vanish at the naked singularity. This non-vanishing is due to the nontrivial contribution of NS-NS $B_{2}$ field. The tension of wrapped D5-brane at the zero-charge locus scales as $T_{F D 3} \sim T_{D 3} g_{s} M$ for a two-sphere $S^{2}\left(\theta_{1}, \phi_{1}\right)$ inside $S^{3}\left(\psi, \theta_{1}, \phi_{1}\right)$. For $S^{2}\left(\theta_{2}, \phi_{2}\right)$ it scales as $T_{F D 3} \sim T_{D 3} g_{s} N / g_{s} M$ which is represented by $T_{D 3} g_{s} M\left(a / \rho_{K}\right)^{2}$. Both tensions are independent of the resolution parameter. Since the $S^{2}\left(\theta_{1}, \phi_{1}\right)$ shrinks to zero size near the apex, the former is rather considered to be the tension of fractional D3brane because a fractional D3-brane is regarded as a D5-brane wrapped over a vanishing 2-cycle. 
Let us turn to the type II supergravity solution corresponding to $M$ wrapped $\mathrm{D}(p+2)$ branes ( fractional D $p$-branes ) and $N$ regular $\mathrm{D} p$-branes on a $(9-p)$-dimensional conical transverse space whose base is an $(8-p)$-dimensional Einstein manifold $X_{8-p}$ [29]. The dilaton, NS-NS 2-form potential and the warp function are expressed by using a radial coordinate $r$ here as

$$
\begin{aligned}
e^{4 \phi} & =h(r)^{3-p}, B_{2}=f \omega_{2}=P \frac{r^{p-3}}{p-3} \omega_{2}, \\
h(r) & =\left(\frac{q}{r}\right)^{7-p}-\frac{P^{2}}{(3-p)(10-2 p) r^{10-2 p}}
\end{aligned}
$$

for $p \neq 3,5$, where $P \sim g_{s} M \alpha^{\prime(5-p) / 2}$ and $q^{7-p} \sim g_{s} N \alpha^{\prime(7-p) / 2}$. The $X_{8-p}$ is assumed to have a 2-cycle associated with a harmonic 2 -form $\omega_{2}$ and the fractional Dp-brane is the $\mathrm{D}(\mathrm{p}+2)$ brane wrapped over the 2-cycle. The $\omega_{2}$ may be normalized such that $\omega_{2} \wedge *_{8-p} \omega_{2}$ is the volume form on $X_{8-p}$. Hereafter we restrict ourselves to $p<3$ where the naked singularity appears at $r=r_{h}$ with $r_{h}^{3-p}=P^{2} /\left((3-p)(10-2 p) q^{7-p}\right)$. We estimate the IR scale $r_{I}$ here simply from $h^{\prime}(r)=0$ as

$$
r_{I}^{3-p}=\frac{P^{2}}{(3-p)(7-p) q^{7-p}} .
$$

From $r_{I}^{3-p}=((10-2 p) /(7-p)) r_{h}^{3-p}$ we note that $r_{I}>r_{h}$ for $p<3$. If we assume that the 2 -cycle is a $S^{2}$, then the tension of wrapped $\mathrm{D}(p+2)$-brane is given by

$$
T_{F D p}=4 \pi T_{D(p+2)} h^{(p-3) / 4}\left(\alpha h r^{4}+f^{2}\right)^{1 / 2},
$$

where there is the contribution of dilaton and $\alpha$ is some constant that depends on $p$ through the embedding of the 2-cycle in $X_{8-p}$. As the tension of fractional $\mathrm{D} p$-brane in the IR we evaluate (37) at $r=r_{I}$ as

$$
T_{F D p} \sim \frac{T_{D p}}{\alpha^{\prime}} P^{(p-1) / 2} r_{I}^{(p-3)^{2} / 2},
$$

where the contribution of NS-NS flux is the same order as the gravitational contribution. Its scaling is expressed as

$$
T_{F D p} \sim T_{D p}\left(g_{s} M\right)^{(p-1) / 2}\left(\frac{r_{I}}{\sqrt{\alpha^{\prime}}}\right)^{(p-3)^{2} / 2},
$$

which is compared with the tension of fractional D3-brane for the resolved conifold that is linear in $M$.

\section{Area law behaviors of Wilson loops}

We investigate the Wilson loop along two space directions in the solution of $N$ D3-branes and $M$ fractional D3-branes on the general resolved conifold in the Euclidean metric. Since the string is stretched in the radial direction, the relevant action in the static gauge for the spatial Wilson loop is given by

$$
S=T T_{F} \int d x \sqrt{h^{-1}+\kappa^{-1}\left(\partial_{x} \rho\right)^{2}}
$$


where $T_{F}=1 / 2 \pi \alpha^{\prime}$ and there is no NS-NS flux contribution for this configuration. From the prescription in Refs. [30, 31], the energy of the string configuration is given by

$$
E=\frac{S}{T}=T_{F} \int_{\rho_{0}}^{\infty} \frac{d \rho}{\sqrt{\kappa\left(1-c_{0}^{2} h\right)}}
$$

since the static solution is represented by $h^{-1} / \sqrt{h^{-1}+\kappa^{-1}\left(\partial_{x} \rho\right)^{2}}=c_{0}$. Owing to $c_{0}^{2}=1 / h\left(y_{0}\right)$ it is further rewritten by

$$
E=\frac{T_{F} a}{2} \int_{y_{0}}^{\infty} \frac{d y}{\sqrt{y \kappa}} \frac{\sqrt{h\left(y_{0}\right)}}{\sqrt{h\left(y_{0}\right)-h(y)}}
$$

where $y=\rho^{2} / a^{2}$ for the resolved conifold. For the general conifold $y=\rho^{2} / b^{2}$ and $a$ in (42) is replaced by $b$. The $y_{0}=\rho_{0}^{2} / a^{2}$ or $\rho_{0}^{2} / b^{2}$ is the minimal value of $y$. The distance $l$ between a quark and an anti-quark is expressed in terms of a parameter $y_{0}$ as

$$
\frac{l}{2}=\frac{a}{2} \int_{y_{0}}^{\infty} \frac{d y}{\sqrt{y \kappa}} \frac{h(y)}{\sqrt{h\left(y_{0}\right)-h(y)}}
$$

We focus on the long distance behavior of the potential energy between a quark and an anti-quark. The main contribution to the integral in (43) is provided by the region near $y=y_{0}$. Since the factor $\left(h\left(y_{0}\right)-h(y)\right)^{1 / 2}$ is approximately expanded by $\left(-\left(y-y_{0}\right) h^{\prime}\left(y_{0}\right)-\right.$ $\left.\left(y-y_{0}\right)^{2} h^{\prime \prime}\left(y_{0}\right) / 2-\cdots\right)^{1 / 2}$ in this region, we take the limit $y_{0} \rightarrow y_{K}=\rho_{K}^{2} / a^{2}$ or $\rho_{K}^{2} / b^{2}$ for the distance $l$ that is a function of $y_{0}$. Then we obtain the logarithmic divergence of $l$ because $h^{\prime}\left(y_{K}\right)$ vanishes. In this limit the main contribution to the integral in (42) also comes from the same region. Combining (43) with (42) we have

$$
E=\frac{1}{4 \pi \alpha^{\prime} \sqrt{h\left(y_{0}\right)}} l+\frac{T_{F} a}{2 \sqrt{h\left(y_{0}\right)}} \int_{y_{0}}^{\infty} \frac{d y}{\sqrt{y \kappa}} \sqrt{h\left(y_{0}\right)-h(y)} .
$$

Hence the first term is leading in the limit $y_{0} \rightarrow y_{K}$ and gives rise to the area law behavior of the Wilson loop. The tension of the confining string is thus derived as

$$
T_{c s}=\frac{1}{4 \pi \alpha^{\prime} \sqrt{h\left(y_{K}\right)}} .
$$

For the resolved conifold the tension is obtained by

$$
T_{c s} \sim \frac{1}{g_{s} M} \frac{\left(g_{s} M\right)^{2} a^{2}}{g_{s} N \alpha^{2}}
$$

which is expressed as

$$
T_{c s} \sim \frac{1}{g_{s} M}\left(\frac{\rho_{K}}{\alpha^{\prime}}\right)^{2} .
$$


This result takes the same form as the tension $\left(\epsilon^{2 / 3} / \alpha^{\prime}\right)^{2} / g_{s} M$ for the deformed conifold [18, 21]. We have again observed the correspondence between $\rho_{K} / \alpha^{\prime}$ and $m$. For the general conifold the tension shows the similar behavior

$$
T_{c s} \sim \frac{1}{g_{s} M} \frac{\left(g_{s} M\right)^{2} b^{2}}{g_{s} N \alpha^{2}} .
$$

Indeed there is a difference between the warp function in the IR region characterized by the logarithmic function for the general conifold and the warp function showing the power behavior for the resolved conifold, but the resulting tensions of the confining string turn out to be the same form where $a$ and $b$ give the scales where non-perturbative effects become relevant in the dual gauge theory. In order to extract the large $l$ behavior we need to take the limit $y_{0} \rightarrow y_{K}$ and observe that the vanishing of derivative of the warp function at the zero-charge locus plays an important role to obtain the area law behavior for the Wilson loop.

\section{Conclusions}

Although there appears the naked singularity in the resolved conifold solution or the general conifold solution of $N$ D3-branes and $M$ fractional D3-branes, we have demonstrated that the IR solution located at the zero-charge locus of the R-R 5-form field, behind which the naked singularity exists, gives the interesting non-perturbative dynamics of its dual $S U(M)$ gauge theory in the confining phase. For the resolved conifold the baryon mass as well as the tension of domain wall at the zero-charge locus are characterized by the resolution parameter through the dimensional transmutation in a similar way that those at the tip $\tau=0$ for the deformed conifold are specified by the deformation parameter. We have evaluated them using the resolved conifold metric itself, whereas the rescaled one of the deformed conifold metric has been used where the scaling is performed in the transverse part of the metric so that its effect is reflected to produce the 4-dimensional mass scale $m$ in the longitudinal part. We have assumed that the resolved conifold solution should not be able to continue to the region where the D3-brane charge is negative, and focused on the IR behavior at the zero-charge locus. Since the resolved conifold is constructed from the conifold by allowing an asymmetry between the two $S^{2}$ parts in the metric we have seen that there are two types of probe configurations associated with the baryon and the domain wall according to which $S^{2}$ is included into the wrapped $S^{3}$. Specially when the D3-brane or the D5-brane wraps $S^{3}$ including the $S^{2}$ with a finite radius near the apex, the $B_{2}$ contribution to the baryon mass and the tension of domain wall is more dominant than the gravitational one. We have also observed that the $B_{2}$ contribution prevents the tension of fractional D3-brane from vanishing at the naked singularity in the resolved conifold and this tension becomes linear in $M$ at the zero-charge locus.

We have shown the area law behavior of the Wilson loop for the $N$ D3-branes and $M$ fractional D3-branes on the resolved or general conifold, and demonstrated that the tension of the confining string takes the same behavior as that for the deformed conifold. The suitable correspondence between the $\rho_{K} / \alpha^{\prime}$ for the resolved conifold and the mass scale $m$ for the deformed conifold has been presented in the baryon mass and the tensions of the domain 
wall and the confining string. In Ref. 26] the behavior of Wilson loop was analyzed for only the $N$ regular D3-branes on the resolved or general conifold. Since there is no zero-charge locus, the distance between a quark and an anti-quark does not exhibit the logarithmic increase. The existence of the zero-charge locus owing to the additional $M$ fractional D3branes yields the logarithmic increase of the distance and hence is important for the area law behavior of the Wilson loop. For the other non-conformal supergravity solutions with less supersymmetry, it would be interesting to study the NS-NS two-form contribution to the tensions of various D-branes wrapped over some cycle associated with the NS-NS flux and ask how the non-conformal property provided by the fractional D-branes is connected with the non-perturbative property in the IR.

\section{References}

[1] J. Maldacena, Adv. Theor. Math. Phys. 2 (1998) 231, hep-th/9711200.

[2] S.S. Gubser, I.R. Klebanov and A.M. Polyakov, Phys. Lett. B428 (1998) 105, hepth/9802109.

[3] E. Witten, Adv. Theor. Math. Phys. 2 (1998) 253, hep-th/9802150.

[4] J. Polchinski and M.J. Strassler, hep-th/0003136.

[5] M.R. Douglas and G. Moore, hep-th/9603167.

[6] S. Kachru and E. Silverstein, Phys. Rev. Lett. 80 (1998) 4855, hep-th/9802183.

[7] A. Lawrence, N. Nekrasov and C. Vafa, Nucl. Phys. B533 (1998) 199, hep-th/9803015.

[8] A. Kehagias, Phys. Lett. B435 (1998) 337, hep-th/9805131.

[9] I.R. Klebanov and E. Witten, Nucl. Phys. B536 (1998) 199, hep-th/9807080.

[10] D.R. Morrison and M.R. Plesser, Adv. Theor. Math. Phys. 3 (1999) 1, hep-th/9810201.

[11] M.R. Douglas, JHEP 9707 (1997) 004, hep-th/9612126.

[12] S.S. Gubser and I.R. Klebanov, Phys. Rev. D58 (1998) 125025, hep-th/9808075.

[13] I.R. Klebanov and N.A. Nekrasov, Nucl. Phys. B574 (2000) 263, hep-th/9911096.

[14] I.R. Klebanov and A.A. Tseytlin, Nucl. Phys. B578 (2000) 123, hep-th/0002159.

[15] J.M. Maldacena and C. Nunez, Phys. Rev. Lett. 86 (2001) 588, hep-th/0008001.

[16] M. Bertolini, P. Di Vecchia, M. Frau, A. Lerda, R. Marotta and I. Pesando, JHEP 0102 (2001) 014, hep-th/0011077; M. Bertolini, P. Di Vecchia, G. Ferretti and R. Marotta, hep-th/0112187.

[17] J. Polchinski, Int. J. Mod. Phys. A16 (2001) 707, hep-th/0011193. 
[18] I.R. Klebanov and M. Strassler, JHEP 0008 (2000) 052, hep-th/0007191.

[19] E. Cáceres and R. Hernández, Phys. Lett. B504 (2001) 64, hep-th/0011204.

[20] A. Loewy and J. Sonnenschein, JHEP 0108 (2001) 007, hep-th/0103163.

[21] C.P. Herzog, I.R. Klebanov and P. Ouyang, hep-th/0108101.

[22] A. Buchel, Nucl. Phys. B600 (2001) 219, hep-th/0011146.

[23] A. Buchel, C.P. Herzog, I.R. Klebanov, L. Pando Zayas and A.A. Tseytlin, JHEP 0104 (2001) 033, hep-th/0102105.

[24] S.S. Gubser, C.P. Herzog, I.R. Klebanov and A.A. Tseytlin, JHEP 0105 (2001) 028, hep-th/0102172.

[25] L.A. Pando Zayas and A.A. Tseytlin, JHEP 0011 (2000) 028, hep-th/0010088.

[26] L.A. Pando Zayas and A.A. Tseytlin, Phys. Phys. D63 (2001) 086006, hep-th/0101043.

[27] Y. Imamura, Prog. Theor. Phys. 100 (1998) 1263, hep-th/9806162; Nucl. Phys. B537 (1999) 184, hep-th/9807179.

[28] A. Brandhuber, N. Itzhaki, J. Sonnenschein and S. Yankielowicz, JHEP 9807 (1998) 020, hep-th/9806158.

[29] C. P. Herzog and I.R. Klebanov, Phys. Rev. D63 (2001) 126005, hep-th/0101020.

[30] J. Maldacena, Phys. Rev. Lett. 80 (1998) 4859, hep-th/9803002.

[31] S.J. Rey and J. Yee, hep-th/9803001; S.J. Rey, S. Theisen and J. Yee, Nucl. Phys. B527 (1998) 171, hep-th/9803135. 